\title{
Endotracheal tube fire during tracheostomy
}

\author{
Eunju Lee, Su-Nam Lee, Jong-il Kim, and Youbin Son \\ Department of Anesthesiology and Pain Medicine, Korea Cancer Center Hospital, Seoul , Korea
}

Despite the reduced use of inflammable anesthetics, there are still a number of reported fire incidents. If an endotracheal tube ignites during anesthesia, it progresses rapidly and the patient experiences a mild to a heavy burn. A severe burn may even cause death [1].

A male patient $(171 \mathrm{~cm}, 51 \mathrm{~kg})$ with a chief complaint of odynophagia was diagnosed with tongue cancer from computed tomography (CT) scan and biopsy. A tracheostomy was scheduled before a radical excision. A brain tumor was discovered by chance from a preoperative magnetic resonance imaging (MRI) scan but it was decided that only observation of the progress would be made because of the very small size of the tumor.

Upon arrival in the operating room patient's oxygen saturation was $95 \%$. Anesthesia induction was done and the anesthesiologist intubated the patient. Using direct curvedblade laryngoscopy, a 7.0 internal diameter reinforced polyvinyl chloride endotracheal tube (ETT; Safety-Flex with Murphy Eye; Mallinckrodt, Athlone) was intubated without difficulty. The tube cuff was filled with air. After intubation, the ETT was fixed at a depth of $22 \mathrm{~cm}$ from the teeth. A normal breathing sound was made from the auscultation of both lungs. Afterwards, anesthesia maintenance was done with sevoflurane (1-2 vol\%, oxygen $2 \mathrm{~L} / \mathrm{min}$ ) and nitrous oxide ( $2 \mathrm{~L} / \mathrm{min})$.

An experienced ear-nose-throat (ENT) surgeon who had performed 40 tracheostomies sterilized the surgical site with betadine and alcohol ( $83 \%$ ethanol). After $10 \mathrm{~min}$, electrocautery in the monopolar cutting mode was used for skin incision at the $2-4^{\text {th }}$ tracheal cartilage, at the midpoint between the cricoids cartilage and the sternal notch. Ten minutes into the surgery, the anesthesiologist discovered that the $\mathrm{ETCO}_{2}$ waves were irregular, so he switched respiration to the manual mode. When observing the surgical view, he discovered that the cuff of the ETT had burst. The ENT surgeon tried to stop the bleeding at the tracheal incision site using electrocautery in the bipolar coagulation mode (25 watt). At that moment, grey smoke coming from the tracheostoma was observed. The authors assessed that it was due to an ignition from the ETT. Manual ventilation and the supply of oxygen, nitrous oxide, and sevoflurane from the anesthesia machine were stopped. Smoke came from the tracheostoma for about $3 \mathrm{sec}$. The ENT surgeon twice poured $10 \mathrm{cc}$ saline at the fire site and extinguished the fire. From the point of ignition to the point of fire extinguishment, the patient was maintained in the state of apnea to reduce the possibility of foreign-body aspiration. At the time of ignition, the tracheostomy had been almost

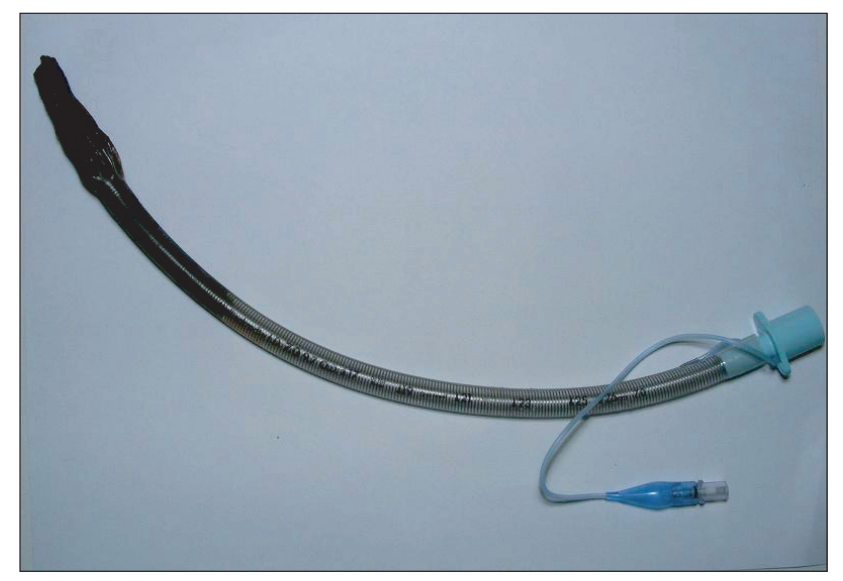

Fig. 1. Endotracheal tube extubated from the patient. Note the carbides are mostly in the cuff.

Corresponding author: Eunju Lee, M.D., Department of Anesthesiology and Pain Medicine, Korea Cancer Center Hospital, 75, Nowon-gil (215-4 Gongneung-dong), Nowon-gu, Seoul 139-706, Korea. Tel: 82-2-970-2820, Fax: 82-2-970-2413, E-mail: ejleemd@hotmail.com

(c) This is an open-access article distributed under the terms of the Creative Commons Attribution Non-Commercial License (http:// creativecommons.org/licenses/by-nc/3.0/), which permits unrestricted non-commercial use, distribution, and reproduction in any medium, provided the original work is properly cited. 
complete. The incinerated ETT was extubated, and another ETT of the same type was inserted in the complete tracheostomy site (Fig. 1). In the process, the patient maintained a 99\% oxygen saturation rate. Total anesthetic time was about $30 \mathrm{~min}$.

The patient was sent to the intensive care unit. Oxygen (5 L/ min) was supplied through the ETT. The patient maintained spontaneous respiration with no respiratory distress or pain. Upon arrival, the oxygen saturation rate was $95 \%$ and $\mathrm{PaO}_{2}$ was $65.8 \mathrm{mmHg}$. Two hours afterwards, the medical team extubated the ETT and supplied oxygen through the tracheostoma.

A bronchoscopy was performed immediately. It showed black carbide covering the area from the trachea to both of the main bronchi. A chest radiograph showed no abnormal findings. Seven weeks afterwards, the patient was moved to the general ward. Oxygen $(2 \mathrm{~L} / \mathrm{min})$ was supplied through a nasal cannula.

Treatment had been planned for the brain tumor. However, the rapid growth and progression of the tumor was fatal before any treatment commenced.

The ignition of the electrocautery-caused ETT fire in this case is considered to be due to not fully considering the possibility of ignition of the ETT, not immediately changing the ETT when the cuff burst, not lowering the oxygen ratio, and continuing with the electrocautery procedure.

In this case, the ignition source was the ETT. It was observed that the cuff of ETT was the most severely charred. The $\mathrm{FiO}_{2}$ of the supplied oxygen was 0.5 . The higher the oxygen rate, the higher is the possibility of fire. If the ratio of oxygen is constant and the ratio of nitrous oxide rises, the risk of a fire rises [2]. The most common fire sources have been reported to be electrocautery (59\%), lasers (32\%), and optical cables (7\%) [3]. Electrocautery can produce temperatures of up to $900-1,500^{\circ} \mathrm{C}$ and most likely cause a fire in the ETT [4].

There may be several preventive measures for fire. At first, inflammables must be removed from the surgical view. In situations with the risk of a fire, using electrocautery must be avoided. In a tracheostomy with the risk of ignition, the oxygen ratio should be lowered and air should be used instead of $\mathrm{N}_{2} \mathrm{O}$. If the trachea is open and the ETT is exposed, the ETT should be covered with a wet gauze to separate the ETT from the electrocautery. Also filling the cuff of the ETT with saline solution may be considered. This method reduces the risk of ignition, but it requires care that the cuff does not burst from external pressure. If a fire occurs, the surgeon and anesthesiologist must quickly communicate with each other and the anesthesiologist must stop patient respiration, temporarily separate the respiratory circuit from the anesthesia machine and remove the remaining heat and oxygen. Afterwards, respiration and anesthesia should be maintained and the damage site should be observed with direct laryngoscopy and bronchoscopy. If there are carbides, they must be removed, and washing the trachea may be required. Severe lung damage from heat or smoke will require long-term airway maintenance and mechanical ventilation, and a high concentration of steroids as a short-term therapy and antibiotics therapy may help [5].

Use of electrocautery facilitates incision and hemostasis, but it can be a source of ignition. Because an endotracheal tube fire may occur from the use of electrocautery, even with the usually delivered oxygen ratio for general anesthesia, the risk of a fire will always be present if precautions towards fire are not taken.

Despite the decline of use of flammable anesthetics in general anesthesia, endotracheal tube fires are possibly occur during a tracheostomy. We report a case of airway fire during a tracheostomy before main surgery. The fire occurred during a surgical tracheostomy under general anesthesia following ignition of the endotracheal tube by diathermy. The endotracheal tube was charred and it's lumen had melted immediately after the accident, bronchofibroscopic examination revealed inhalation injury. We discuss factors that increase the risk of airway fire and management steps that should be undertaken in the event that such a fire occurs.

\section{References}

1. Niskanen M, Purhonen S, Koljonen V, Ronkainen A, Hirvonen E. Fatal inhalation injury caused by airway fire during tracheostomy. Acta Anaesthesiol Scand 2007; 51: 509-13.

2. Wolf GL, Simpson JI. Flammability of endotracheal tubes in oxygen and nitrous oxide enriched atmosphere. Anesthesiology 1987; 67: 236-9.

3. Smith LP, Roy S. Operating room fires in otolaryngology: risk factors and prevention. Am J Otolarlyngol 2011; 32: 109-14.

4. Bowdle TA, Glenn M, Colston H, Eisele D. Fire following use of electrocautery during emergency percutaneous transtracheal ventilation. Anesthesiology 1987; 66: 697-8.

5. Miller RD. Miller's Anesthesia. 7th ed. Philadelphia, Churchill Livingston. 2009, p 2416. 